\title{
The ionization of galaxies by their planetary nebulae
}

\author{
Grażyna Stasińska \\ LUTH, Observatoire de Paris, CNRS, Université Paris Diderot; Place Jules Janssen 92190 \\ Meudon, France \\ email: grazyna.stasinsa@obspm.fr
}

\begin{abstract}
Recent studies have shown that nuclei of planetary nebulae and their remnants (dubbed HOLMES for "hot low-mass evolved stars" ) can easily explain two long-standing problems of extragalactic astronomy: the observed emission-line spectra of ellipticals and LINER-like galaxies and the ionization and heating of the diffuse interstellar medium in spirals. They are summarized in this contribution. It is emphasized that the computation of grids of stellar evolution models until the white dwarf stage is essential not only for the study of planetary nebulae but also for the study of the ionization of galaxies.
\end{abstract}

Keywords. Planetary nebulae: general, stars: AGB and post-AGB, galaxies: general

\section{Introduction}

Studies dealing with planetary nebulae generally focus either on individual objects, or on their collective use as test particles to probe the AGB nucleosynthesis, the chemical evolution of galaxies or their dynamics. Apart from the well known studies on the planetary nebulae luminosity function (Jacoby et al. 1999, but see also Marigo et al. 2004 and Ciardullo et al. 2005) very few studies have been devoted to the entire population of planetary nebulae. It appears that the population of planetary nebulae and related objects plays a role on a galactic scale that has been fully recognized only recently . As a matter of fact, the title of this paper is somewhat incorrect, because what it really presents is the role of hot low-mass evolved stars (HOLMES) in the ionization of galaxies, of which central stars of planetary nebulae represent only a fraction.

In this contribution I summarize some work on this topic in which I participated.

\section{Cases where the ionization in galaxies need explanation}

\subsection{Elliptical Galaxies}

These are red, passive galaxies that have stopped forming stars. They were formerly considered to be devoid of gas and dust. However, deep imaging and spectroscopy starting from the mid nineties revealed the presence of a multiphase interstellar medium (ISM) that includes ionized gas.

\subsection{Low ionization nuclear emission line regions (LINERs)}

They were discovered by Heckman (1980) and found to be present in nearly $40 \%$ of nearby galaxies (Ho 2008). Then, with the advent of the Sloan Digital Sky Survey (SDSS, York et al. 2000), which produced spectra of nearly one million of galaxies, it was found that galaxies with integrated LINER-like spectra compose a large part of the entire population of galaxies in the nearby Universe(Kauffmann et al. 2003). The source of ionization of 
LINERs has been a subject of debate since their discovery. The presence of nuclear activity, similar to that of Seyfert galaxies but at a much lower level was among the first suggestions, competing with that of ionizing shocks.

\subsection{The diffuse ionized gas outside the disks of spiral galaxies (DIG)}

Its existence was discovered in the seventies. Many different ionizing sources have been invoked, such as ionizing photons from leaking H II regions located in the disk, shocks, dissipation of turbulence, magnetic reconnection etc... (see Haffner et al. 2009 for a recent review).

These three different classes of objects share the common property that they are all found in the LINER zone of the famous $[\mathrm{O} \mathrm{III}] / \mathrm{H} \beta$ versus $[\mathrm{NII}] / \mathrm{H} \alpha$ diagram which is commonly used to infer the main ionizing source of ionized nebulae or galaxies (Baldwin, Phillips \& Terlevich, 1981, referred to as BPT). This implies that the gas in these objects is ionized by a radiation field harder than that from massive OB stars which ionize normal $\mathrm{H}$ II regions or pure star-forming galaxies (see e.g. Stasińska 2007 for a basic interpretation of the BPT diagram).

These three different classes are known to contain old stellar populations, which produce planetary nebulae. On average, planetary nebulae have central stars with higher effective temperatures than massive OB stars. This immediately suggests that planetary nebulae may play a role in the ionization of the objects mentioned above. Perhaps the reason why such a suggestion was rarely considered in the past is that the total number of planetary nebulae that are detected in galaxies is not very large, and certainly cannot explain the ionization in elliptical galaxies or in the extraplanar gas of spiral galaxies. But detected planetary nebulae represent just the tip of the iceberg, being the brightest planetary nebulae present in those galaxies. One also has to consider the radiation field from fainter planetary nebulae, as well as from "naked" hot white dwarfs. All those taken together compose the population of HOLMES. In the following, we consider the population of HOLMES predicted by theoretical models.

\section{Ionizing photons from a coeval stellar population}

During the first few $10^{6} \mathrm{yr}$ after an instantaneous burst of star formation, massive stars produce about $10^{47} \mathrm{~s}^{-1}$ ionizing photons per solar mass. This number steadily decreases with the gradual death of the most massive stars. When the last massive supernova has exploded, roughly after $10^{8} \mathrm{yr}$, ionizing photons start being produced by the hot phases of evolved low-and intermediate-mass stars, the HOLMES. Their integrated production rate is by about 5 orders of magnitude smaller than that of the massive stars, but it is roughly constant during a Hubble time (see Fig. 2 in Cid Fernandes et al. 2011). This is because main sequence lifetimes of stars that go through the HOLMES phase span the entire Hubble time. The hardness of the ionizing photons produced by the HOLMES is much higher than that of massive stars, and is also roughly constant over a Hubble time. Therefore, in any zone in a galaxy that happens to be devoid of massive stars HOLMES provide a steady floor of hard ionizing photons. The question which remains is whether the HOLMES are numerous enough to explain the observed emission line luminosities. 


\section{Explaining the ionization in galaxies with HOLMES}

\subsection{Elliptical Galaxies}

The first model explaining the emission lines in elliptical galaxies was proposed by Binette et al. (1994) who computed the Lyman continuum output of an ageing starburst and found that HOLMES can explain both the observed equivalent width of hydrogen lines and the observed emission-line ratios. But observational constraints were then scarce.

\subsection{LINER-like galaxies}

A revolution in the observations came with the SDSS which obtained fiber-fed spectra in the $3900-9500 \AA$ range for nearly one million galaxies. Analysis of these spectra with inverse spectral synthesis tools (such as STARLIGHT by Cid Fernandes et al. 2005) was as second revolution. The decomposition of the complex stellar populations of galaxies into simple stellar populations of various ages and metallicities allows one to determine the star formation and chemical history of galaxies and, simultaneously, to dig out weak emission lines from stellar emission. Applying STARLIGHT to the SDSS galaxies, Stasińska et al. (2008) found that galaxies that have LINER-like spectra, have stopped forming stars and contain old stellar populations whose HOLMES can easily produce the emission-line ratios observed in these galaxies. The observed $\mathrm{H} \alpha$ luminosities can be accounted by the HOLMES in about $1 / 3$ of the LINER-like galaxies present in the BPT diagram. So, the population of LINER-like galaxies is composed in part of "retired" galaxies, i.e. galaxies that stopped froming stars. However, we know (from their X-ray emission or radio spectra or line profiles) that LINER-like galaxies with weakly active galactic nuclei (AGN) also exist. So, how to, at least statistically, distinguish both kinds of objects just from their optical spectra? This cannot be done using the BPT diagram, since both families occupy the same region of the BPT plane. Another weakness of the BPT diagram is that it requires four lines to be measured, which leaves aside a large fraction of galaxies with weak emission lines. In Cid Fernandes et al. (2010) we proposed to use another diagram which requires only the two strongest lines and plots the $\mathrm{H} \alpha$ equivalent width, $\mathrm{W}(\mathrm{H} \alpha)$, as a function of $[\mathrm{N} \mathrm{II}] / \mathrm{H} \alpha$ (the WHAN diagram). Using this diagram, in Cid Fernandes et al. (2011) we could identify five spectral classes in the entire population of galaxies:

- Pure star forming galaxies: $\log [\mathrm{N} \mathrm{II}] / \mathrm{H} \alpha<-0.4$ and $W(\mathrm{H} \alpha)>3 \AA$

- Strong AGN (i.e., Seyferts): $\log [\mathrm{N} \mathrm{II}] / \mathrm{H} \alpha>-0.4$ and $W(\mathrm{H} \alpha)>6 \AA$

- Weak AGN: $\log [\mathrm{N} \mathrm{II}] / \mathrm{H} \alpha>-0.4$ and $3<W(\mathrm{H} \alpha)<6 \AA$

- Retired galaxies (i.e., fake AGN): $W(\mathrm{H} \alpha)<3 \AA$

- Passive galaxies (actually, line-less galaxies): $W(\mathrm{H} \alpha)$ and $W([\mathrm{~N} \mathrm{II}])<0.5 \AA$

A comparative analysis of star formation histories and of other properties of galaxies in these different classes corroborated our proposed differentiation between retired galaxies and weak AGN in the LINER-like family.

Integral field spectroscopy of LINER-like galaxies has now confirmed that in many of them ionization is indeed due to HOLMES and not to a weak AGN (Annibali et al. 2010, Sarzi et al. 2010).

\subsection{The DIG in spiral galaxies}

In spiral galaxies, the main ionizing source consists of massive OB stars distributed in the thin disk. The DIG is detected at several kiloparsecs above the galactic plane. While leakage of $\mathrm{OB}$ stellar photons out of the $\mathrm{H}$ II regions may indeed contribute to ionize the DIG, it is not sufficient to explain all its observed properties, in particular the increase of $[\mathrm{N} \mathrm{II}] / \mathrm{H} \alpha,[\mathrm{S} \mathrm{II}] / \mathrm{H} \alpha,[\mathrm{O} \mathrm{II}] / \mathrm{H} \beta$ and $[\mathrm{O} \mathrm{III}] / \mathrm{H} \beta$ with galactic height. HOLMES, which 
are plentiful in the thick discs and lower haloes of spiral galaxies, have a radiation field roughly similar to that of a $10^{5} \mathrm{~K}$ star and are thus an obvious candidate for the ionization and heating of the DIG. Taking the edge-on spiral galaxy NGC 891 as a test case, we have shown in Flores-Fajardo et al. (2011) that the estimated content of HOLMES in this galaxy well explains the observed emission-line characteristics of its extraplanar DIG.

\section{Summary and prospects}

HOLMES, thus, provide a good and natural explanation to the problem of heating and ionization of gas in galaxies where star formation has stopped as well as in the diffuse ionized medium of star-forming galaxies. Their expected population yields a sufficient number of ionizing photons to produce detectable emission lines and the hardness of their radiation field is able to produce LINER-like line ratios. Until recently, HOLMES were broadly ignored in problems dealing with ionization in galaxies, while, in absence of massive stars, they should not.

However, it is fair to remark that, quantitatively, the results above depend on the availability of theoretical tracks for stellar evolution of low and intermediate mass stars until the white dwarf stage. The computation of such tracks is notoriously difficult, especially if one needs to reach until after the TP-AGB, and the parameter space to explore is large (initial masses, metallicities, rotation etc...). However, such a work is essential not only for many studies on planetary nebulae, as has been shown during this symposium, but also for the understanding of the ionization of galaxies on a large scale.

\section{Acknowledgements}

I am indebted to my colleagues from the SEAGAL-STARLIGHT project, in particular R. Cid Fernandes, N. Vale Asari, A. Mateus from UFSC (Brazil) as well as to C. Morisset, N. Flores-Fajardo and L. Binette from UNAM (Mexico) for a wonderful collaboration. I also thank the SOC of this symposium on planetary nebulae for financial support and for allowing me to give this presentation.

\section{References}

Annibali, F., et al., 2010, A\&SA, 519, A40

Baldwin, J. A., Phillips, M. M., \& Terlevich, R., 1981, PASP, 93, 5

Binette, L., Magris, C. G., Stasińska, G., \& Bruzual, A. G., 1994, A\& A, 292, 13

Ciardullo, R., Sigurdsson, S., Feldmeier, J. J., \& Jacoby, G. H., 2005, AIPC, 804, 292

Cid Fernandes, R., Mateus, A., Sodré, L., Stasińska, G., \& Gomes, J. M., 2005, MNRAS, 358, 363

Cid Fernandes, R., Stasińska, G., Schlickmann, M. S., Mateus, A., Vale Asari, N., Schoenell, W., \& Sodré, L., 2010, MNRAS, 403, 1036

Cid Fernandes, R., Stasińska, G., Mateus, A., \& Vale Asari, N., 2011, MNRAS, 413, 1687

Flores-Fajardo, N., Morisset, C., Stasińska, G., \& Binette, L., 2011, MNRAS, 415, 2182

Haffner, L. M., et al., 2009, RvMP, 81, 969

Heckman, T. M., 1980, A\& $A, 87,152$

Ho, L. C., 2008, ARA $\mathscr{S} A, 46,475$

Jacoby, G. H., Ciardullo, R., \& Feldmeier, J. J., 1999, ASPC, 167, 175

Kauffmann, G., et al., 2003, MNRAS, 346, 1055

Kewley, L. J., Groves, B., Kauffmann, G., \& Heckman, T., 2006, MNRAS, 372, 961

Marigo, P., Girardi, L., Weiss, A., Groenewegen, M. A. T., \& Chiosi, C., 2004, A\&A, 423, 995

Sarzi, M., et al., 2010, MNRAS, 402, 2187

Stasińska, G., 2007, arXiv, arXiv:0704.0348

Stasińska, G., et al., 2008, MNRAS, 391, L29

Weiss, A., \& Ferguson, J. W., 2009, A\&A, 508, 1343

York, D. G., et al., 2000, AJ, 120, 1579 